\title{
Locally optimal designs for the simple death process
}

\author{
D.E. Pagendam* P.K. Pollett ${ }^{\dagger}$
}

8th April 2010

\begin{abstract}
We demonstrate the existence and uniqueness of optimal observation schedules for discrete observation of the simple death process. Optimality is framed in the sense of observing the process at times that maximise the Fisher information for the death rate. We examine the relationship of our designs with those obtained by other authors for the simple birth process. A number of interesting properties are uncovered. Practical considerations in the application of these designs in an experimental setting are investigated, and we examine the performance of some approximately optimal designs that are usually simpler to implement. We will show that our optimal designs are highly robust to mis-specification of the death rate.
\end{abstract}

Keywords: Markov chain, optimal design, Fisher information, sampling, observation, death process

\section{Introduction}

Very little attention has been given to experimental design where the underlying model involves the observation of a Markov process over time. One exception is the paper by Becker \& Kersting (1983), which establishes the existence and uniqueness of a locally optimal design for the simple birth process. Using a similar approach to theirs, we study two related design problems for the simple death process. Experiments involving death processes arise in the study of disease dynamics, for example where organisms are exposed to pathogens in a controlled environment and monitored over time in order to estimate parameters such as infection rates and death rates (Kleczkowski \& Gilligan 2007, Cook, Gibson \& Gilligan 2008).

Experimental designs for the simple death process have previously been considered by Chernoff (1953), Ehrenfeld (1962) and, more recently, by Cook et al. (2008). However, the question of the existence and uniqueness of an optimal design, and specification of its exact form, was not resolved by these authors except in the most simple single observation case (Chernoff 1953). Additionally, these authors limited their attention to one particular type of design, which we refer to here as the time-unconstrained design, where there is no upper limit on the time available to observe the process. Ogawa (1962) derived a timeunconstrained design, which was optimal in the sense that $k$ order statistics of independent

*Department of Mathematics, University of Queensland, Qld 4072 Australia. pagendam@maths.uq.edu.au

${ }^{\dagger}$ Department of Mathematics, University of Queensland, Qld 4072 Australia. pkp@maths.uq.edu.au 
and identically distributed exponential random variables were chosen in order to construct the best linear unbiased estimator for the rate parameter. There is also a connection with the design of dilution experiments of the form outlined by Fisher (1922), and highlighted briefly by Ford, Titterington \& Kitsos (1989). We also discuss an otherwise neglected design, which we refer to as the time-constrained design, where there is a given time interval in which all observations must be taken. We show that this is closely related to Becker \& Kersting's (1983) design for the simple birth process. Additionally we explain how to devise optimal or approximately optimal designs, again a subject which has received little attention. Ehrenfeld (1962) suggested that in many experimental contexts, taking observations at equidistant times is sufficiently close to optimal for practical purposes. Whilst this may be true in some circumstances, successful use of the equidistant design hinges on the choice of a suitable stopping rule for taking the last observation; we address the important issue of how an appropriate stopping rule can be identified.

We consider the simple death process, which is a discrete-state Markov process $(Y(t), t \geq$ $0)$ taking non-negative integer values, with initial state $Y(0)=y_{0}(>0)$ and infinitesimal transition probabilities

$$
\operatorname{Pr}\{Y(t+h)=n+m \mid Y(t)=n\}= \begin{cases}1-n \mu h+o(h) & \text { if } m=0 \\ n \mu h+o(h) & \text { if } m=-1 \\ o(h) & \text { otherwise }\end{cases}
$$

where the single parameter $\mu(>0)$ represents the per-capita death rate of organisms. In an experimental context, this process provides a useful statistical model for a cohort of organisms with independent and identically distributed exponential lifetimes, and $Y(t)$ counts the number to have survived by time $t$. A realisation of the simple death process is presented in Figure 1 (a). This model is applicable, for example, to epidemiological and survival analysis experiments where organisms are subject to some toxin or disease such that the hazard rate is constant. Whilst more general hazard functions will not be considered in detail, we will see that our designs can be adapted to handle power-law hazard functions.

Much attention has been given to estimating parameters of birth-death processes where observation is continuous and therefore all the information about the parameters is available; see (Basawa \& Prakasa Rao 1980) for a good overview. However, in most practical situations, such as the monitoring of a population or cohort of organisms, the process cannot be observed continuously and the researcher must make discrete observations. Moreover, there is usually some constraint limiting the number of opportunities to observe the process. Our aim here is to devise an optimal observation schedule $\boldsymbol{t}=\left(t_{1}, \ldots, t_{n}\right)$, comprising a series of observations $\boldsymbol{y}=\left(y_{1}, \ldots, y_{n}\right)$ of the simple death process, that maximises the Fisher information about the value of the per-capita death rate $\mu$. This type of design can be thought of as a one dimensional version of the well known D-optimal design (Box \& Lucas 1959, Atkinson \& Donev 1992), which aims to minimize the width of confidence intervals for $\mu$. We assume that the initial state $y_{0}$, at time $t_{0}=0$, is known and that we are limited to $n$ observations of the process. We consider two cases: (i) where there is no time constraint and (ii) where we have a fixed time interval $\left[0, t_{\max }\right]$ in which to take all observations. In case (i) we specify a unique optimal design, the last observation being taken optimally at $t_{o p t}=t_{n}$. In case (ii), 

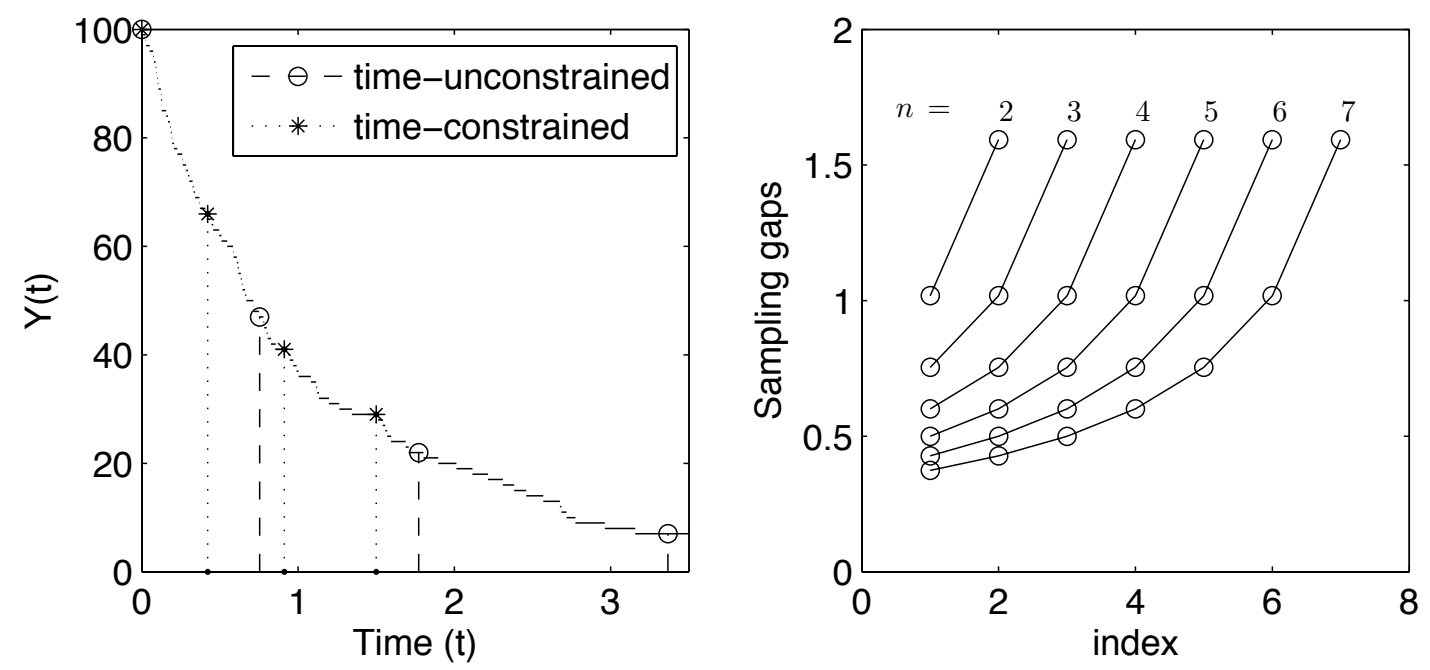

Figure 1: (a) A simulation of the simple death process with $\mu=1$, showing the time unconstrained optimal observation times with $n=3$ and the time constrained optimal observation times with $n=3$ and $t_{\max }=1.5$. (b) Relationship between the optimal observation gaps and the gap indices for designs with varying numbers of observations $(\mu=1)$.

we are again able to specify an optimal design uniquely, but it does not necessarily span the entire interval $\left[0, t_{\max }\right]$. It does if $t_{\text {opt }} \geq t_{\max }$, in which case we show that it is optimal to take the last observation at $t_{n}=t_{\max }$. In contrast, Becker and Kersting constrained their last observation to take place at $t_{n}=t_{\max }$. This is perhaps not unexpected, for with every population increase the process divulges more information. However, our design problem has the added complication that if too much time is allocated between successive observations, the process may reach the absorbing state too early, whence no more information can be obtained. Indeed, we will see that if $t_{\text {opt }}<t_{\max }$ the optimal schedule does not consume the entire period available.

\section{The locally optimal design}

Since there is only one parameter, the optimal design $\boldsymbol{t}^{\star}$ is defined simply to be $\boldsymbol{t}^{\star}=$ $\operatorname{argmax}_{\boldsymbol{t} \in T} \mathcal{I}(\boldsymbol{t})$, where $\mathcal{I}$ is the Fisher information and $T$ is the design space consisting of elements $\boldsymbol{t}=\left(t_{1}, \ldots, t_{n}\right)$ such that $0<t_{1}<\cdots<t_{n}$, and $t_{n} \leq t_{\max }$ in the timeconstrained case. The Fisher information is $\mathcal{I}=-\mathrm{E}\left(\partial^{2} \ell / \partial \mu^{2}\right)$, where $\ell$ is the log-likelihood. For a Markov process $(Y(t), t \geq 0)$, the log-likelihood has a very simple form: denoting the transition probabilities by $p\left(y_{i}, t_{i} \mid y_{i-1}, t_{i-1}\right)=\operatorname{Pr}\left\{Y\left(t_{i}\right)=y_{i} \mid Y\left(t_{i-1}\right)=y_{i-1}\right\}$, we have $\ell(\mu ; \boldsymbol{t}, \boldsymbol{y})=\sum_{i=1}^{n} \log p\left(y_{i}, t_{i} \mid y_{i-1}, t_{i-1}\right)$, remembering that the initial state $y_{0}$ is fixed at time $t_{0}=0$. We consider two experimental scenarios as design problems: first where the practitioner has ample time, and is therefore only constrained by the number of observations $n$, and, second, where all $n$ observations must be scheduled in a fixed interval $\left[0, t_{\max }\right]$. As previ- 
ously indicated, we refer to these as the time-unconstrained and time-constrained schedules, respectively.

The transition probabilities of the simple death process can be exhibited explicitly as

$$
\operatorname{Pr}\{Y(s+t)=y \mid Y(s)=z\}=\left(\begin{array}{l}
z \\
y
\end{array}\right)\{R(t)\}^{y}\{1-R(t)\}^{z-y} \quad(0 \leq y \leq z, s, t \geq 0),
$$

where $R(t)=\exp (-\mu t)$. If the hazard rate $\mu(t)$ were not constant, we would have $R(t)=$ $\exp \left(-\int_{0}^{t} \mu(u) d u\right)$. The log-likelihood is thus given by

$$
\ell(\mu ; \boldsymbol{t}, \boldsymbol{y})=\sum_{i=1}^{n} \log \left(\begin{array}{c}
y_{i-1} \\
y_{i}
\end{array}\right)-y_{i} \mu \tau_{i}+\left(y_{i-1}-y_{i}\right) \log \left\{1-\exp \left(-\mu \tau_{i}\right)\right\},
$$

where $\tau_{i}=t_{i}-t_{i-1}(i=1, \ldots, n)$ are the gaps between successive observations. Clearly $\ell$ is well defined over $T$, for in particular $\tau_{i}>0$ for all $i$. Using the fact that $\mathrm{E}\{Y(t)\}=y_{0} R(t)=$ $y_{0} \exp (-\mu t)$, the Fisher information $\mathcal{I}$ can be evaluated as

$$
\mathcal{I}(\mu, \boldsymbol{t})=y_{0} \sum_{i=1}^{n} \frac{\tau_{i}^{2} \exp \left(-\mu \sum_{j=1}^{i} \tau_{j}\right)}{1-\exp \left(-\mu \tau_{i}\right)}
$$

again well defined over $T$. We identify the optimal schedule by maximizing the Fisher information $\mathcal{I}$ over $\boldsymbol{t}=\left(t_{1}, \ldots, t_{n}\right)$ in the design space. Since this can be done by equivalently maximizing $\mathcal{I}$ over strictly positive $\boldsymbol{\tau}=\left(\tau_{1}, \ldots, \tau_{n}\right)$, we will henceforth take $\mathcal{I}$ to be a function of $\tau$.

It is important to realize that the optimal design will necessarily depend on the parameter $\mu$ that we wish to estimate. Chernoff (1953) referred to designs like these, which depend on the model parameters, as locally optimal. This is a common issue for optimal design in the context of non-linear models. In practice one would use either a best guess at the true parameter value or exploit some prior information about the parameter (Pronzato \& Walter 1985).

\subsection{Time-unconstrained optimal schedule}

Notice that $\mathcal{I}$ is strictly positive in $T$. We will show that $\mathcal{I}$ has a unique stationary point, and that this is a strict local maximum. Since $\mathcal{I}$ is continuous inside $T$ and continuous near its boundary, this must in fact be a global maximum.

Proposition 1 For a simple death process, $\mathcal{I}$ achieves a unique global maximum within the design space. The gaps $\tau_{1}, \ldots, \tau_{n}$ that maximise $\mathcal{I}$ satisfy $0<\tau_{1}<\tau_{2}<\cdots<\tau_{n}$.

Proof. Differentiating $\mathcal{I}$ gives $n$ equations, $\partial \mathcal{I} / \partial \tau_{1}=0, \ldots, \partial \mathcal{I} / \partial \tau_{n}=0$, that are satisfied by any stationary point $\boldsymbol{\tau}=\left(\tau_{1}, \ldots, \tau_{n}\right)$ of $\mathcal{I}$. The last of these reduces to

$$
2-\mu \tau_{n}-2 \exp \left(-\mu \tau_{n}\right)=0 \quad\left(\tau_{n}>0\right)
$$


and, since the left-hand side is obviously monotonically decreasing in $\tau_{n}$, its solution is unique. The solution can be expressed in terms of the principal branch of the Lambert W-function which is herein denoted $W_{0}(\cdot)$ using the convention adopted in (Corless, Gonnett, Hare, Jeffrey \& Knuth 1996):

$$
\tau_{n}=\frac{W_{0}\{-2 \exp (-2)\}+2}{\mu} \simeq 1.5936 / \mu \text {. }
$$

Observe that $\tau_{n}$ is the same for all $n$; it depends only on $\mu$.

The remaining equations reduce to

$$
\frac{\tau_{i}\left\{2-\mu \tau_{i}-2 \exp \left(-\mu \tau_{i}\right)\right\}}{\left\{1-\exp \left(-\mu \tau_{i}\right)\right\}^{2}}=\mu K_{i} \quad(i=1, \ldots, n-1),
$$

where $K_{i}=\sum_{q=i+1}^{n}\left(\tau_{q}^{2} \exp \left(-\mu \sum_{k=i+1}^{q} \tau_{k}\right)\right) /\left(1-\exp \left(-\mu \tau_{q}\right)\right)$. Since $K_{i}$ depends only on $\tau_{i+1}, \ldots, \tau_{n}$, we can solve this system iteratively: for $\tau_{n-1}$ in terms of $\tau_{n}$, then $\tau_{n-2}$ in terms of $\tau_{n-1}$ and $\tau_{n}$, and so forth. Furthermore, the solution at each stage is unique and $\tau_{1}<\tau_{2}<\cdots<\tau_{n}$. To see this, observe that the left-hand side of (4), as a function of $\tau$, that is, $f(\tau):=(\tau\{2-\mu \tau-2 \exp (-\mu \tau)\}) /\left(\{1-\exp (-\mu \tau)\}^{2}\right)(\tau>0)$ decreases strictly from its right-hand limit $1 / \mu$ near $\tau=0$, to 0 at $\tau=\tau_{n}$ as given by (3). So, we must prove that the successive values of $K_{i}$ satisfy $K_{n-1}<K_{n-2}<\cdots<K_{1}<1 / \mu^{2}$. First, from (2) and (3), $K_{n-1}=\tau_{n}\left(2 / \mu-\tau_{n}\right)\left(\simeq 0.6476 / \mu^{2}\right)<1 / \mu^{2}$. Next, for $i=2, \ldots, n-1$,

$$
K_{i-1}=\frac{\tau_{i}^{2} \exp \left(-\mu \tau_{i}\right)}{1-\exp \left(-\mu \tau_{i}\right)}+\exp \left(-\mu \tau_{i}\right) K_{i}
$$

Therefore $K_{i}<1 / \mu^{2}$ implies $K_{i-1}<1 / \mu^{2}$, because $g(\tau):=\exp (-\mu \tau)+\left(\mu^{2} \tau^{2} \exp (-\mu \tau)\right) /(1-$ $\exp (-\mu \tau))(\tau>0)$ has supremum 1 (near $\tau=0)$. We deduce that $K_{i}<1 / \mu^{2}$ for all $i$, and hence that there is a unique stationary point of $\mathcal{I}$ in $T$.

Next we show that the gaps are strictly increasing. For $i=2, \ldots, n-1$,

$$
K_{i-1}-K_{i}=\frac{\tau_{i}^{2} \exp \left(-\mu \tau_{i}\right)}{1-\exp \left(-\mu \tau_{i}\right)}-\left\{1-\exp \left(-\mu \tau_{i}\right)\right\} K_{i}
$$

But, from (4),

$$
K_{i}=\frac{\tau_{i}\left\{2-\mu \tau_{i}-2 \exp \left(-\mu \tau_{i}\right)\right\}}{\mu\left\{1-\exp \left(-\mu \tau_{i}\right)\right\}^{2}}=\frac{\tau_{i}^{2} \exp \left(-\mu \tau_{i}\right)}{\left\{1-\exp \left(-\mu \tau_{i}\right)\right\}^{2}}\left\{\left(\frac{2}{\mu \tau_{i}}-1\right) \exp \left(\mu \tau_{i}\right)-\frac{2}{\mu \tau_{i}}\right\} .
$$

Therefore,

$$
K_{i-1}-K_{i}=\frac{\tau_{i}^{2} \exp \left(-\mu \tau_{i}\right)}{\left\{1-\exp \left(-\mu \tau_{i}\right)\right\}^{2}} \frac{h\left(\mu \tau_{i}\right)}{\mu \tau_{i}}
$$

where $h(x)=x+2+(x-2) \exp (x)$. It is easy to see that $h(x)>0$ for all $x>0$. Therefore, $K_{1}>K_{2}>\cdots>K_{n-1}$, which from (4) implies that $\tau_{1}<\tau_{2}<\cdots<\tau_{n}$. 
To complete the proof we will show that the unique stationary point is a local (and hence global) maximum. The entries of the Hessian $\mathbf{H}$ of $\mathcal{I}$ are easy to evaluate. We find that

$$
\frac{\partial^{2} \mathcal{I}}{\partial \tau_{i} \partial \tau_{j}}=-\mu \frac{\partial \mathcal{I}}{\partial \tau_{\max (i, j)}} \quad(j \neq i)
$$

which is clearly 0 at the stationary point, while the diagonal entries are given by

$$
\frac{\partial^{2} \mathcal{I}}{\partial \tau_{i}^{2}}=2 y_{0} \exp \left(-\mu \sum_{q=1}^{i} \tau_{q}\right) \frac{\left\{\mu \tau_{i}-1+\exp \left(-\mu \tau_{i}\right)\right\}\left\{\mu \tau_{i} \exp \left(-\mu \tau_{i}\right)-1+\exp \left(-\mu \tau_{i}\right)\right\}}{\left\{1-\exp \left(-\mu \tau_{i}\right)\right\}^{3}},
$$

for $i=1, \ldots, n-1$, which is always strictly negative, and

$\frac{\partial^{2} \mathcal{I}}{\partial \tau_{n}^{2}}=\frac{y_{0} \exp \left(-\mu \sum_{q=1}^{n} \tau_{q}\right)}{\left\{1-\exp \left(-\mu \tau_{n}\right)\right\}^{3}} 2\left\{1-\exp \left(-\mu \tau_{n}\right)\right\}\left[\left\{1-\exp \left(-\mu \tau_{n}\right)\right\}-2 \tau_{n} \mu\right]+\tau_{n}^{2} \mu^{2}\left\{1+\exp \left(-\mu \tau_{n}\right)\right\}$,

which is strictly negative at the stationary point (because $\exp \left(-\mu \tau_{n}\right)=2-\mu \tau_{n}$ and $\mu \tau_{n} \simeq$ 1.5936). It follows that $\mathbf{H}$ is negative definite at the stationary point.

Once the gaps $\tau_{1}, \ldots, \tau_{n}$ have been evaluated using (2) and (4), the optimal observation times $t_{1}, \ldots, t_{n}$ can be recovered by setting $t_{j}=\sum_{i=1}^{j} \tau_{i}$. Then, $t_{\text {opt }}=t_{n}$ gives the time over which observations are to be made. If the time $t_{\max }$ available to the practitioner is less than $t_{\text {opt }}$, then one should resort to the time-constrained optimal schedule to be outlined in the next section.

We complete this section by looking at some interesting properties of our design. First, since $\tau_{n} \simeq 1.5936 / \mu$ depends only on $\mu$, successive values $\tau_{n-1}, \tau_{n-2}, \ldots$, determined by (4), also depend only on $\mu$. Thus the effect on the optimal design of increasing $n$ by 1 is to retain the last $n$ gaps and add a single new smallest gap; this is illustrated in Figure 1 (b). Or, in terms of the optimal observation times, if $\boldsymbol{t}^{(n)}=\left(t_{1}^{(n)}, \ldots, t_{n}^{(n)}\right)$ is the $n$-observation time-unconstrained optimal design, then, if $m>n, \boldsymbol{t}^{(m)}=\left(t_{1}^{(m)}, \ldots, t_{m-n}^{(m)}, t_{m-n}^{(m)}+\boldsymbol{t}^{(n)}\right)$, so that the last $n$ times in the $m$-observation optimal design are the $n$-observation optimal times shifted by $t_{m-n}^{(m)}$.

Next, it is easy to verify, by way of (4) and (5), that the optimal gaps satisfy $\varphi_{1}\left(-\mu \tau_{i}\right)=$ $\varphi_{2}\left(-\mu \tau_{i+1}\right)$, for $i=1, \ldots, n-1$, where

$$
\begin{aligned}
& \varphi_{1}(x)=x\{\exp (x)-1\}^{-2}\{2 \exp (x)-x-2\} \text { and } \\
& \varphi_{2}(x)=x \exp (x)\{\exp (x)-1\}^{-2}\{2 \exp (x)-2-x \exp (x)\}
\end{aligned}
$$

because $\varphi_{1}\left(-\mu \tau_{i}\right)=\mu^{2} K_{i}$ and $\varphi_{1}(x)-x^{2}\{\exp (x)-1\}^{-1}=\exp (-x) \varphi_{2}(x)$ for all $x \neq 0$. These are precisely the equations (7) and (8) on Page 259 of Becker \& Kersting (1983) that were used to define the constrained optimal observation scheme for the simple birth process with $t_{n}$ fixed. Since $\varphi_{1}(-x)=\varphi_{2}(x)$ for all $x \neq 0$, we have $\varphi_{1}\left(\mu \tau_{i+1}\right)=\varphi_{2}\left(\mu \tau_{i}\right)$. Conversely, any collection of positive numbers $\tau_{1}, \ldots, \tau_{n-1}$ that satisfy $\varphi_{1}\left(\mu \tau_{i+1}\right)=\varphi_{2}\left(\mu \tau_{i}\right)$ also satisfy (4). This has some interesting consequences. For example, we see there is no loss of generality in taking $\mu=1$; since the optimal design is defined uniquely by $\varphi_{2}\left(\mu \tau_{n}\right)\left(=\varphi_{1}\left(-\mu \tau_{n}\right)\right)=0$ 
and $\varphi_{2}\left(\mu \tau_{i}\right)=\varphi_{1}\left(\mu \tau_{i+1}\right)$ for $i=1, \ldots, n-1$, it is clear that the optimal schedule $\boldsymbol{t}$ can be obtained from the optimal schedule $\overline{\boldsymbol{t}}$ with $\mu=1$ by setting $\boldsymbol{t}=\overline{\boldsymbol{t}} / \mu$. Also, we can determine the optimal gaps explicitly in terms of the Lambert $\mathrm{W}$-function $W_{0}(\cdot)$, as our next result demonstrates. Thus the optimal design can be evaluated simply using any mathematical package capable of calculating $W_{0}(\cdot)$.

Proposition 2 Suppose that $\mu=1$. Let $q_{1}, q_{2}, \ldots, q_{n}$ be the collection of positive numbers defined by $q_{n}=2+W_{0}\{-2 \exp (-2)\}$ and, for $i=1, \ldots, n-1, q_{i}=2+W_{0}\left\{-q_{i+1} \exp \left(-q_{i+1}\right)\right\}$. Then, the optimal gaps $\tau_{1}, \ldots, \tau_{n}$ are given by $\tau_{n}=q_{n}$ and, for $i=1, \ldots, n-1$,

$$
\tau_{i}=q_{i+1}+W_{0}\left\{-q_{i+1} \exp \left(-q_{i+1}\right)\right\} .
$$

Proof. Let $\tau_{n}$ be the solution to (2) with $\mu=1$. Then, from (3), $\tau_{n}=2+W_{0}\{-2 \exp (-2)\}=$ $q_{n}$. Next, it is easy to show that

$$
\varphi_{2}(y)-\varphi_{1}(x)=\frac{[y \exp (y)\{\exp (x)-1\}-x\{\exp (y)-1\}][\exp (y)(q(x)-y)-q(x)]}{\{\exp (x)-1\}\{\exp (y)-1\}^{2}},
$$

for $x, y \neq 0$, where $q(x)=\{\exp (x)-1\}^{-1}\{2 \exp (x)-2-x\}$. The denominator is clearly strictly positive for $x, y>0$, and it is easy to verify that the first factor of the numerator is strictly positive in this region. Therefore, $\varphi_{2}(y)=\varphi_{1}(x)$ if and only if $\exp (y)\{q(x)-y\}-$ $q(x)=0$, which implies that $y=q(x)+W_{0}[-q(x) \exp \{-q(x)\}]$. But $\varphi_{2}\left(\tau_{i}\right)=\varphi_{1}\left(\tau_{i+1}\right)$ for $i=1, \ldots, n-1$, and so $(9)$ holds with $q_{i+1}=q\left(\tau_{i+1}\right)$. Finally, substituting (9) into $q_{i}=q\left(\tau_{i}\right)$, and using the identity $W_{0}(x) \exp \left\{W_{0}(x)\right\}=x$, gives $q_{i}=2+W_{0}\left(-q_{i+1} \exp \left(-q_{i+1}\right)\right)$.

Notice that the function $q$ increases monotonically from $q(0+)=1$ to its limit 2 as $x \rightarrow$ $\infty$. Therefore its inverse is well defined and we may write $\tau_{i}=q^{-1}\left(q_{i}\right)$. The connection with Becker and Kersting's design for the simple birth process will be examined further and exploited in the next section.

\subsection{Time-constrained optimal schedule}

For the time-unconstrained scenario, we have shown that the optimal observation period $\left[0, t_{o p t}\right]$ for the simple death process is dictated solely by the per-capita death rate $\mu$ and the number of observations $n$. However, it is possible that the time available for the experiment will be less than $t_{\text {opt }}$.

Suppose we wish to observe the process $n$ times over a given interval $\left[0, t_{\max }\right]$ and we know the state $y_{0}$ of the process at $t_{0}=0$. In order to determine the optimal schedule we will follow the same programme as above, but subject to the constraint $\sum_{i=1}^{n} \tau_{i} \leq t_{\max }$. We employ the Kuhn-Tucker conditions (Kuhn \& Tucker 1951): for $\boldsymbol{\tau}=\left(\tau_{1}, \ldots, \tau_{n}\right)$ to be a local maximum of $\mathcal{I}$, subject to the constraint, it is necessary that $\partial \mathcal{I} / \partial \tau_{i}-\lambda=0(i=1, \ldots, n)$ and $\lambda\left(\sum_{i=1}^{n} \tau_{i}-t_{\max }\right)=0$, for some $\lambda \geq 0$ (in addition to the constraint), implying that either (i) $\lambda=0$ and $\partial \mathcal{I} / \partial \tau_{i}=0$ for all $i$, or, (ii) $\partial \mathcal{I} / \partial \tau_{1}=\cdots=\partial \mathcal{I} / \partial \tau_{n}=\lambda$, for some strictly positive $\lambda$, and $\sum_{i=1}^{n} \tau_{i}=t_{\max }$. Clearly (i) can happen only when $t_{\max } \geq t_{\text {opt }}$, in which case 
$\boldsymbol{\tau}$ is the unique stationary point obtained in the unconstrained case. Indeed if $t_{\max }>t_{\text {opt }}$ the unconstrained-optimum lies inside the design space of the constrained problem and hence it is the unique global maximum. We will show that when $t_{\max } \leq t_{\text {opt }}$, there is a unique $\tau$ satisfying (ii). This will be done by first identifying a relationship between our optimisation problem and the one considered by Becker \& Kersting (1983) for the simple birth process with $t_{n}=t_{\max }$ fixed. We will then establish that $\boldsymbol{\tau}$ is indeed a global maximum. We begin with the following simple observation, which follows immediately from (ii).

Proposition 3 If $t_{\max } \leq t_{\text {opt }}$, and there is an optimal schedule $\left(t_{1}, \ldots, t_{n}\right)$, then $t_{n}=t_{\max }$.

Thus, as one might have expected, it is optimal to take the last observation at $t_{\max }$. Our next result identifies the unique solution to our optimisation problem with $t_{0}=0$. It is identical to Becker \& Kersting's (1983) solution for the simple birth process over $\left[0, t_{\max }\right]$, but with the gaps in reverse order. This is perhaps not surprising, because, sample path for sample path, those paths of the simple birth process that start at 1 and finish at $i(\geq 1)$ have the same likelihood as reverse paths of simple death process starting at $i$ and finishing at 1 . But note that there is a chance that the death process will reach 0 within the period $\left[0, t_{\max }\right]$, yet there is no corresponding reverse sample path in the birth process.

Proposition 4 There is a unique optimal schedule $\left(t_{0}^{*}, t_{1}^{*}, \ldots, t_{n}^{*}\right)$ with $t_{0}=0$. If $t_{\text {max }}>t_{\text {opt }}$ it is the one identified in Proposition 1. If $t_{\max } \leq t_{\text {opt }}$ it is the time-reverse of the corresponding optimal schedule $\left(t_{0}^{\star}, t_{1}^{\star}, \ldots, t_{n}^{\star}\right)$ over $\left[0, t_{\max }\right]$ for the simple birth process with per-capita birth rate $\mu$, in that $t_{j}^{*}=t_{\max }-t_{n-j}^{\star}$ for all $j$.

Proof. We have already dealt with the case $t_{\max }>t_{\text {opt }}$, so assume that $t_{\text {max }} \leq t_{\text {opt }}$ and suppose that $\boldsymbol{\tau}=\left(\tau_{1}, \ldots, \tau_{n}\right)$ is a local maximum of $\mathcal{I}$ subject to the constraint $\sum_{i=1}^{n} \tau_{i} \leq t_{\max }$. Then, since (ii) must hold for some $\lambda>0$, it is necessary that $\partial \mathcal{I} / \partial \tau_{i}=\partial \mathcal{I} / \partial \tau_{i+1}(i=1, \ldots, n-1)$, which, on setting $x_{i}=-\mu \tau_{i}$, becomes

$$
\frac{x_{i}\left\{2 \exp \left(x_{i}\right)-x_{i}-2\right\}}{\left\{\exp \left(x_{i}\right)-1\right\}^{2}}=\frac{x_{i+1} \exp \left(x_{i+1}\right)\left\{2 \exp \left(x_{i+1}\right)-2-x_{i+1} \exp \left(x_{i+1}\right)\right\}}{\left\{\exp \left(x_{i+1}\right)-1\right\}^{2}} .
$$

Thus, $\boldsymbol{\tau}$ must satisfy $\varphi_{1}\left(-\mu \tau_{i}\right)=\varphi_{2}\left(-\mu \tau_{i+1}\right)$, and hence $\varphi_{1}\left(\mu \tau_{i+1}\right)=\varphi_{2}\left(\mu \tau_{i}\right)$, where $\varphi_{1}$ and $\varphi_{2}$ are given by (8).

Now, from the proof of Theorem 1 of Becker \& Kersting (1983), for any given $t>0$ there is a unique collection of non-negative numbers, $x_{1}, x_{2}, \ldots, x_{n}$, with sum equal to $t$ that satisfy $\varphi_{1}\left(x_{i}\right)=\varphi_{2}\left(x_{i+1}\right)(i=1, \ldots, n-1)$; this is also a consequence of Proposition 2. It follows immediately that $\varphi_{1}\left(\mu \tau_{i+1}\right)=\varphi_{2}\left(\mu \tau_{i}\right)$ has a unique non-negative solution $\tau^{*}=\left(\tau_{1}^{*}, \ldots, \tau_{n}^{*}\right)$ satisfying $\sum_{i=1}^{n} \tau_{i}=t_{\max }$, which can be found by setting $t=\mu t_{\max }$ and $\tau_{i}^{*}=x_{n+1-i} / \mu$. In particular, our local maximum is unique and equal to $\tau^{*}$. Moreover, from Theorem 1 of Becker \& Kersting (1983), it is clear that $\tau_{i}^{*}=\tau_{n+1-i}^{\star}$, where $\left(\tau_{1}^{\star}, \ldots, \tau_{n}^{\star}\right)$ are the gaps corresponding to the optimal observation schedule for the birth process with per-capita birth rate $\mu$ over the period $\left[0, t_{\max }\right]$. Hence, the corresponding observation times satisfy

$$
t_{j}^{*}=\sum_{i=1}^{j} \tau_{i}^{*}=\sum_{i=1}^{j} \tau_{n+1-i}^{\star}=\sum_{i=n+1-j}^{n} \tau_{i}^{\star}=\sum_{i=1}^{n} \tau_{i}^{\star}-\sum_{i=1}^{n-j} \tau_{i}^{\star}=t_{m a x}-t_{n-j}^{\star} .
$$


It remains to prove that $\tau^{*}$ is a indeed a local maximum. This is easy to establish here, because the Hessian of the Lagrangian $\mathcal{L}(\boldsymbol{\tau})=\mathcal{I}(\boldsymbol{\tau})-\lambda\left(\sum_{i=1}^{n} \tau_{i}-t_{\max }\right)$ is equal to the Hessian $\mathbf{H}$ of $\mathcal{I}(\boldsymbol{\tau})$, evaluated earlier. We must show that $\mathbf{H}\left(\boldsymbol{\tau}^{*}\right)$ is negative definite on the tangent plane at $\boldsymbol{\tau}^{*}$ on the surface $S$ determined by any active constraints. For our problem this is simple, because there is a single active constraint: $S$ is the hyperplane $\left\{\boldsymbol{\tau} \in \mathbb{R}^{n}\right.$ : $\left.\sum_{i=1}^{n} \tau_{i}=t_{\text {max }}\right\}$ and the tangent plane is $\left\{\boldsymbol{y} \in \mathbb{R}^{n}: \sum_{i=1}^{n} y_{i}=0\right\}$. It is therefore sufficient to check that $\boldsymbol{y} \mathbf{H}\left(\boldsymbol{\tau}^{*}\right) \boldsymbol{y}^{\prime}<0$ for all $\boldsymbol{y} \in \mathbb{R}^{n}$ with $\boldsymbol{y} \neq \mathbf{0}$ and $\sum_{i=1}^{n} y_{i}=0$. This is equivalent to checking that $\overline{\mathbf{H}}\left(\boldsymbol{\tau}^{*}\right)$, obtained from $\mathbf{H}\left(\boldsymbol{\tau}^{*}\right)$ by deleting the last (the $n$-th) row and column, is negative definite over $\mathbb{R}^{n-1}$. But this follows from the proof of Proposition 1: because $\tau_{1}^{*}, \ldots, \tau_{n-1}^{*}$ satisfy (4), it is clear from (6) and (7) that $\mathbf{H}\left(\boldsymbol{\tau}^{*}\right)$ is negative definite over $\mathbb{R}^{n-1}$.

The relationship between the unconstrained and constrained designs is now transparently clear. For both designs the first $n-1$ optimal gaps $\tau_{1}^{*}, \ldots, \tau_{n-1}^{*}$ are the same function of $\tau_{n}^{*}$, and can be evaluated in terms of $\tau_{n}^{*}$ from $\varphi_{2}\left(\mu \tau_{i}\right)=\varphi_{1}\left(\mu \tau_{i+1}\right)$ (equivalently (4)). For the unconstrained problem $\tau_{n}^{*}$ is determined by (3). The resulting observation schedule will also be optimal for the constrained problem if $t_{\text {opt }}<t_{\max }$; otherwise the condition $\sum_{i=1}^{n} \tau_{i}=t_{\max }$ is used, in place of (4), to determine the optimal gaps. Proposition 2 provides a means of evaluating the unconstrained optimal design. For the constrained problem with $t_{\text {opt }} \geq t_{\max }$, Proposition 2 can be used in conjunction with, say, a bisection search, at each stage checking whether $t_{\max }-\sum_{i=1}^{n} \tau_{i}$ is sufficiently small. This latter case is illustrated in Figure 1 (a) along with a simulation of the death process.

Furthermore, because of the connection with Becker and Kersting's design for the simple birth process with per-capita birth rate $\mu$, many of the analytical properties identified by those authors carry over to our design with obvious modification. For example, if we refer to equation (12) of Becker \& Kersting (1983), there is no loss of generality in taking $\mu=1$ or equivalently, no loss of generality in taking $t_{\max }=1$. To obtain the optimal schedule $\boldsymbol{t}$ over $\left[0, t_{\max }\right]$ we calculate the $\mu=1$ optimal schedule $\overline{\boldsymbol{t}}$, but over $\left[0, \mu t_{\max }\right]$, and then set $\boldsymbol{t}=\overline{\boldsymbol{t}} / \mu$.

\section{Comparing designs}

Here we examine the information loss between the optimal design and the continuous observation of the process, and then compare it to two equidistant designs using two stopping rules. Optimal designs were obtained using a bisection algorithm.

\subsection{Comparison with continuous observation}

Consider the scenario where the simple death process is observed continuously over a fixed interval of time $\left[0, t_{\max }\right]$, so that the exact times $x_{i}$ between successive deaths are recorded. Let $t_{k}=\sum_{i=1}^{k} x_{i}$ denote the time of the last death observed by $t_{\max }$. The likelihood is

$$
L(\mu ; \boldsymbol{x}, k)=\exp \left\{-\mu\left(y_{0}-k\right)\left(t_{\max }-t_{k}\right)\right\} \prod_{i=1}^{k}\left(y_{0}-i+1\right) \mu \exp \left\{\left(y_{0}-i+1\right) \mu x_{i}\right\}
$$


and the expected Fisher information is $\mathcal{I}_{\text {cont }}=y_{0} \mu^{-2}\left\{1-\exp \left(-\mu t_{\text {max }}\right)\right\}$. So, the efficiency of an $n$-observation design $\left(t_{1}, \ldots, t_{n}\right)$ relative to its continuous counterpart is

$$
\frac{\mathcal{I}_{n}}{\mathcal{I}_{\text {cont }}}=\frac{\mu^{2}}{1-\exp \left(-\mu t_{\text {max }}\right)} \sum_{i=1}^{n} \frac{\tau_{i}^{2} \exp \left(-\mu \sum_{j=1}^{i} \tau_{j}\right)}{1-\exp \left(-\mu \tau_{i}\right)}
$$

Denote the Fisher information for the $n$-observation optimal design by $\mathcal{I}_{\text {opt }}$. Figure 2 (a) illustrates the performance of this design relative to continuous observation for various values of $\mu t_{\max }$ and $n$. For any given $n$ the point $\left(\mu t_{\max }=\mu t_{\text {opt }}\right)$ where the solid curve becomes a dashed curve is the point above which the unconstrained design is also optimal for the constrained problem. We see that, for any given $\mu$, when $t_{\max }$ is small there is little information loss for small numbers of observations, but that the information loss increases substantially with $t_{\max }$ for small numbers of observations. For large $t_{\max }$ the information loss stabilises as the limiting value $\ell_{n}$ of the relative efficiency can be exhibited explicitly from (11) as $\ell_{n}=\sum_{i=1}^{n} g_{i}^{2}\left\{1-\exp \left(-g_{i}\right)\right\}^{-1} \exp \left(-\sum_{j=1}^{i} g_{j}\right)$, where $g_{i}=\phi\left(q_{i}\right)$, given in Proposition 2, are the $\mu=1$ optimal gaps. This is not surprising, for the process yields less and less information about $\mu$ with time, eventually giving no more once the process is absorbed. It is also evident that for larger numbers of observations there is much less information loss. Indeed if we fix $t_{\max }$ and let $n$ get large, the contribution of additional observations to the total Fisher information diminishes, again perhaps not surprising, because in the limit our optimal observation is effectively continuous observation. Overall, our analyses demonstrate that in an experimental context, choosing the number of observation opportunities should be carefully weighed against the costs and benefits, since a relatively small number of observations may perform almost as well as a large number of observations.

\subsection{Comparison with equidistant designs}

We now examine the efficiency of some equidistant designs relative to the optimal design. The first, which we call the naïve equidistant design, is constructed by making observations that are equally spaced on the whole interval $\left[0, t_{\max }\right]$. The second, which we call the optimal equidistant design, has equally spaced observations over an optimal observation interval $\left[0, t_{e q}\right]$, with a gap size $\tau$ that maximizes the Fisher information

$$
\mathcal{I}=y_{0}\left(\tau^{2} \exp (-\mu \tau)\{1-\exp (-n \mu \tau)\}\right) /\left(\{1-\exp (-\mu \tau)\}^{2}\right)
$$

with $\mathcal{I}_{\text {naive }}$ and $\mathcal{I}_{\text {eq }}$ denoting the maximal Fisher information for these designs, Figures 2 (b) and (c) show the efficiency of each relative to the optimal design for various values of $\mu t_{\text {max }}$ and $n$. (Note that when $n=1$, the optimal equidistant design and the optimal design are identical.) It will be clear that an equidistant design can perform almost as well as the optimal design, as suggested in (Ehrenfeld 1962), but that this hinges on the choice of an appropriate stopping rule. Indeed the stopping rule is more important than the actual timing of the observations. When $t_{\max }$ is large the naïve design performs poorly, and very poorly when $t_{\max }>t_{\text {eq }}$, yet the optimal equidistant design performs well even for small $n$. 


\subsection{Comparison with logarithmic designs}

Finally, we compare the optimal design with what we will call the optimal logarithmic design. A logarithmic design has observation times $t_{1}, \ldots, t_{n}$ chosen so that per-capita expected number of deaths on the interval $\left(t_{i-1}, t_{i}\right]$ is the same for all $i$, and hence $t_{i}=-(1 / \mu) \log b_{i}$, where $b_{i}=1-\frac{i}{n}\left(1-\exp \left(-\mu t_{n}\right)\right)$. The optimal logarithmic design is obtained by choosing $t_{n}$ so as to maximize the Fisher information

$$
\mathcal{I}=\frac{y_{0} n}{\mu^{2}\left(1-\exp \left(-\mu t_{n}\right)\right)} \sum_{i=1}^{n} b_{i} b_{i-1} \log ^{2}\left(b_{i} / b_{i-1}\right) .
$$
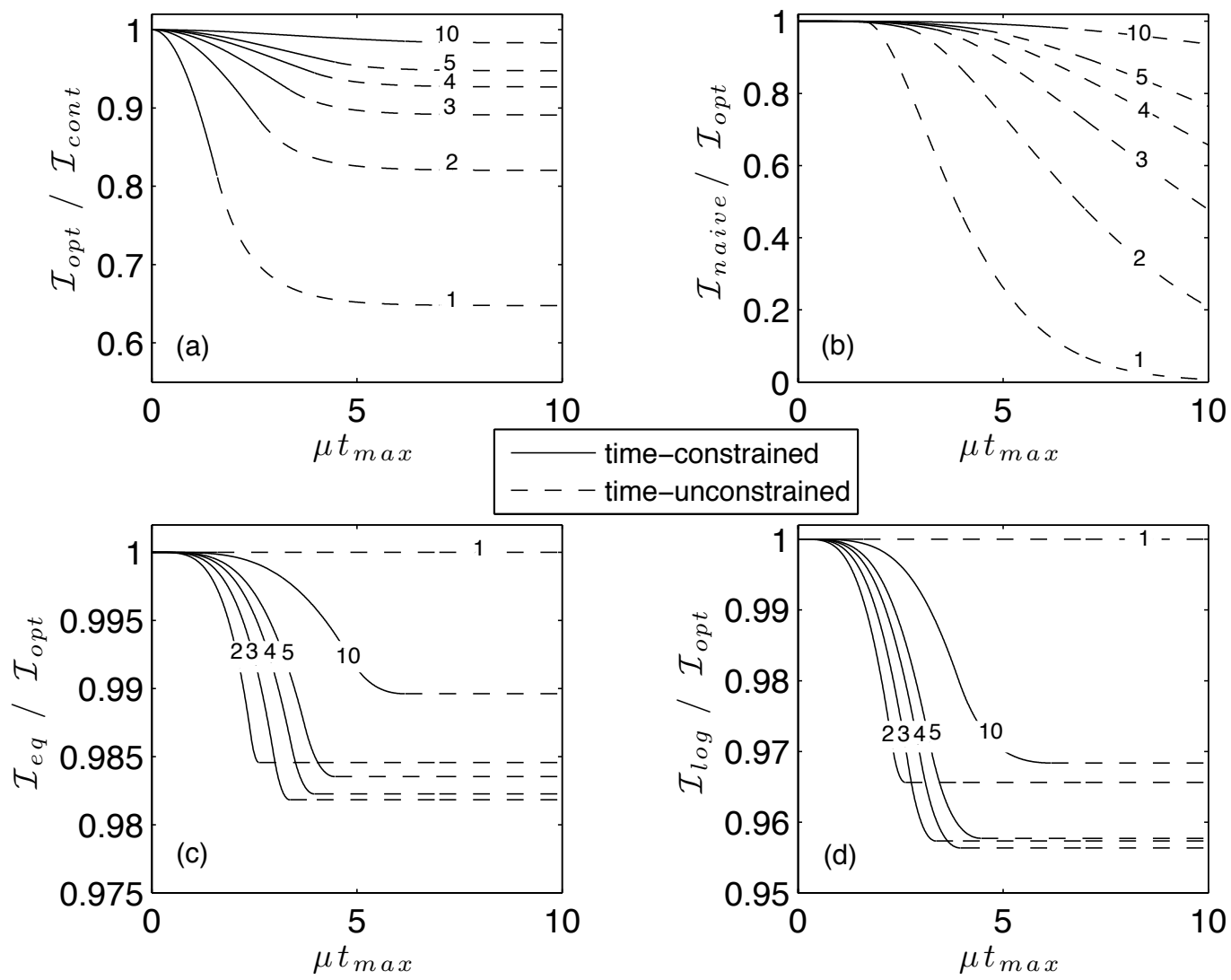

Figure 2: Relative Efficiencies of designs for various values of $\mu t_{\max }$ and $n$ (labelled on the curves): (a) optimal design relative to continuous observation; (b) naïve equidistant design relative to the optimal design; (c) optimal equidistant design relative to the optimal design; and (d) optimal logarithmic design relative to the optimal design.

Figure 2 (d) illustrates the performance of the optimal logarithmic design relative to the optimal design. Again note that, when $n=1$, the optimal logarithmic design is identical to 
the optimal design. The curves in this figure show a strong resemblance to those in Figure 2 (c). However, it is notable, and perhaps surprising, that there is slightly greater information loss relative to the optimal design compared with the optimal equidistant design. Despite the poorer performance, the optimal logarithmic design performs extremely well, capturing at worst more that $95 \%$ of the information of the optimal design.

\section{Robustness}

Finally, we examine the robustness of our designs to mis-specification of the death rate parameter $\mu$. Suppose that $\mu_{\text {true }}$ is the true value of $\mu$ and that $\mu_{\text {guess }}$ is the value used to determine the optimal schedule. Correspondingly, let $\mathcal{I}_{\text {true }}$ be the Fisher Information about $\mu_{\text {true }}$ using the schedule that is optimal for $\mu_{\text {true }}$ and let $\mathcal{I}_{\text {guess }}$ be the Fisher Information about $\mu_{\text {true }}$ using the schedule that is optimal for $\mu_{\text {guess }}$. We will evaluate the relative change $R=\left(\mathcal{I}_{\text {guess }}-\mathcal{I}_{\text {true }}\right) / \mathcal{I}_{\text {true }}(\leq 0)$ in terms of $\epsilon=\left(\mu_{\text {guess }}-\mu_{\text {true }}\right) / \mu_{\text {true }}$, the relative error in our guessing $\mu_{\text {true }}$ to be $\mu_{\text {guess }}$.

First consider the unconstrained case. Recall that for a given $\mu$ the optimal schedule $\boldsymbol{t}$ can be obtained from the $\mu=1$ optimal schedule $\overline{\boldsymbol{t}}$ by setting $\boldsymbol{t}=\overline{\boldsymbol{t}} / \mu$. Since, from (1), $\mathcal{I}(\mu, \boldsymbol{t})=\mathcal{I}(1, \mu \boldsymbol{t}) / \mu^{2}$, it is easy to see that $R=\mathcal{I}(1, r \overline{\boldsymbol{t}}) / \mathcal{I}(1, \overline{\boldsymbol{t}})-1$, where $r=\mu_{\text {true }} / \mu_{\text {guess }}=$ $(1+\epsilon)^{-1}$. Furthermore, it is clear that $R=R(\epsilon)$ has a power series expansion near $\epsilon=0$, and, since $R(0)=R^{\prime}(0)=0, R(\epsilon)=K_{n} \epsilon^{2}+\circ\left(\epsilon^{2}\right)$, where $K_{n}$ depends only on the number of observations $n$. Values of $K_{n}$ for $n=1, \ldots, 10$ are given in Table 1. Figure 3 (a) illustrates the relative change in efficiency as $\epsilon$ is allowed to vary, and Table 2 gives the relative decrease for a mis-specification of $\mu$ of up to 20 percent in the case of $n=5$ observations. We see that the optimal design is highly robust to mis-specification of $\mu$ and that over specifying $\mu$ will lead to a more robust design.

\begin{tabular}{c|cccccccccc}
$n$ & 1 & 2 & 3 & 4 & 5 & 6 & 7 & 8 & 9 & 10 \\
\hline$K_{n}$ & 0.2968 & 0.3302 & 0.1776 & 0.1099 & 0.0755 & 0.0550 & 0.0428 & 0.0342 & 0.0280 & 0.0234
\end{tabular}

Table 1. Second order terms $K_{n}$ in the power series expansion for $R(\epsilon)$.

\begin{tabular}{c|ccccccccc}
$\epsilon(\%)$ & -20 & -15 & -10 & -5 & 0 & 5 & 10 & 15 & 20 \\
\hline Decrease in FI $(\%)$ & 0.56 & 0.30 & 0.13 & 0.03 & 0 & 0.03 & 0.12 & 0.25 & 0.44
\end{tabular}

Table 2. Relative decrease in the Fisher Information for different values of $\epsilon(n=5)$.

In the case where $\boldsymbol{t}$ is constrained to be in $\left[0, t_{\text {max }}\right]$, we can again set $\boldsymbol{t}=\overline{\boldsymbol{t}} / \mu$, but now $\overline{\boldsymbol{t}}$ is the $\mu=1$ optimal schedule constrained to be in $\left[0, \mu t_{\max }\right]$. We find that $R=$ $\mathcal{I}\left(1, r \overline{\boldsymbol{t}}_{\text {guess }}\right) / \mathcal{I}\left(1, \overline{\boldsymbol{t}}_{\text {true }}\right)-1$, where $\overline{\boldsymbol{t}}_{\text {guess }}$ and $\overline{\boldsymbol{t}}_{\text {true }}$ are the $\mu=1$ optimal designs constrained to be in $\left[0, \mu_{\text {guess }} t_{\text {max }}\right]$ and $\left[0, \mu_{\text {true }} t_{\text {max }}\right]$, respectively. As before, $R=R(\epsilon)$ has a power series expansion near $\epsilon=0$ and $R(\epsilon)=K_{n} \epsilon^{2}+\circ\left(\epsilon^{2}\right)$, but now $K_{n}$ will also depend on $t_{\text {max }}$. Figure 3 
(a)
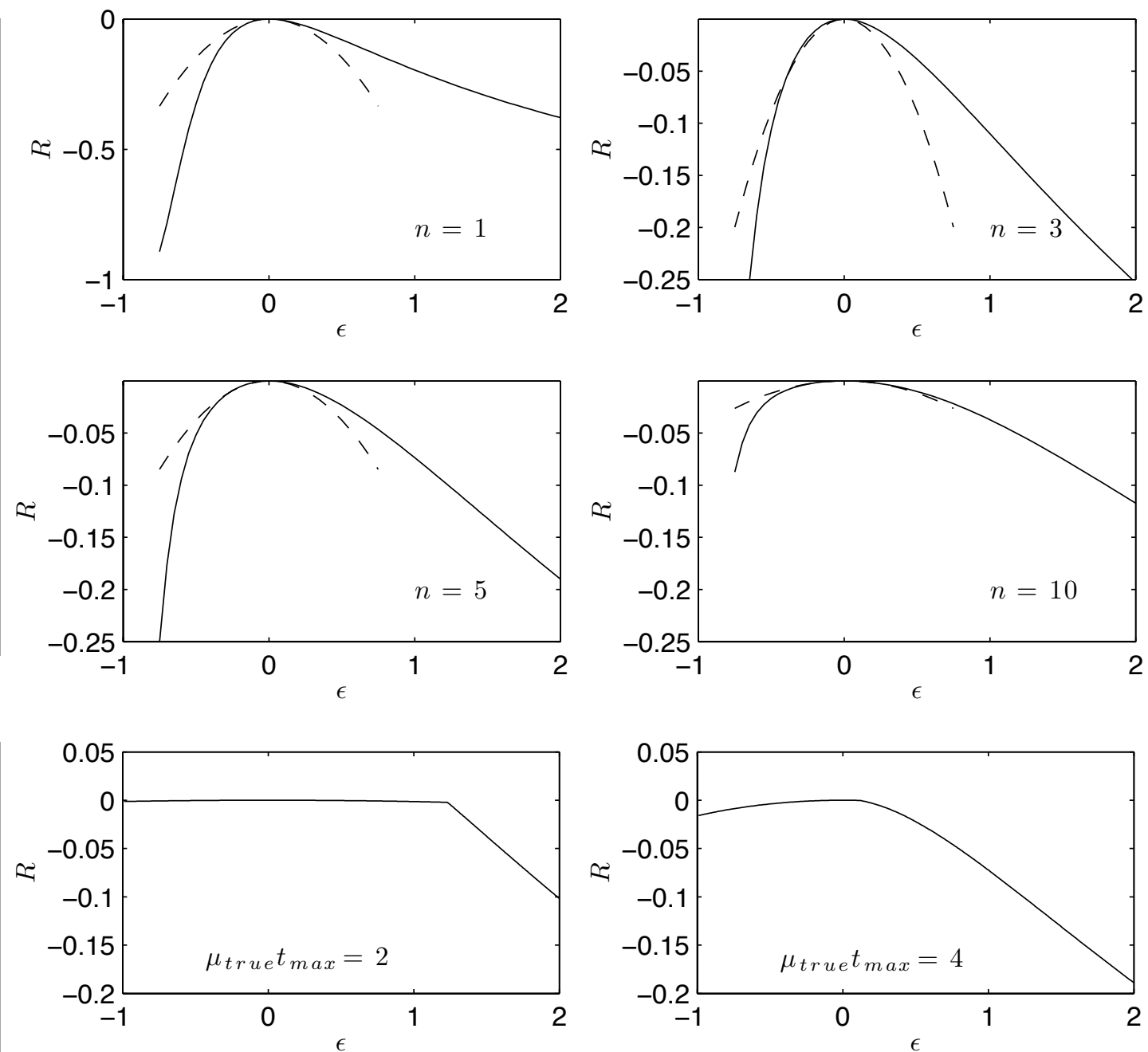

(b)
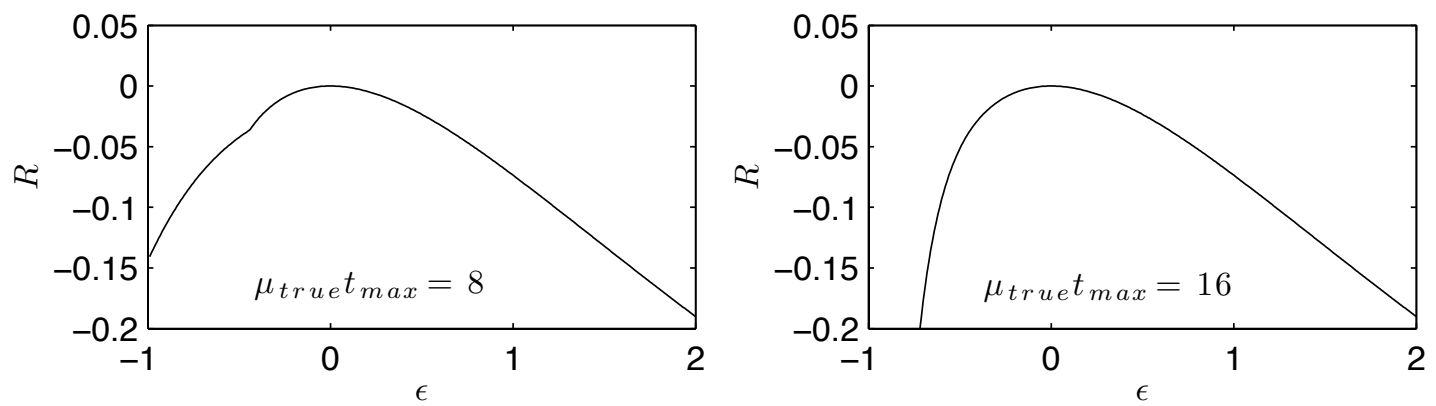

Figure 3: The relative change in efficiency against the relative error $\epsilon$ : (a) unconstrained case for $n=1,3,5$ and 10 observations; and (b) the constrained case for $n=5$ and $\mu_{\text {true }} t_{\text {max }}=2$, 4,8 and 16. The solid line is the true profile. The dashed line is the approximation using the second order perturbation expansion.

(b) illustrates the relative change in efficiency in the case of $n=5$ observations. Notice that when $\mu_{\text {true }} t_{\max }$ is large the profile of relative change in efficiency is similar to that depicted 
in Figure 3 (a) for $n=5$. This is expected. When $\mu_{\text {true }} t_{\text {max }}=16$ both optimal designs are effectively unconstrained in that they do not occupy the whole of $\left[0, t_{\text {max }}\right]$, while the kink in the $\mu_{\text {true }} t_{\text {max }}=8$ profile corresponds to the point below which the design based on $\mu_{\text {guess }}$ always occupies the whole of $\left[0, t_{\max }\right]$. We also see that when $\mu$ is under specified the constrained design is generally more robust than the corresponding unconstrained design; if $\mu_{\text {guess }}$ is too small we are penalized by less because the observations occupy a smaller interval than they would if the design were unconstrained.

\section{Conclusion}

We have established the existence and uniqueness of locally optimal designs for the simple death process, and showed that they are highly robust to mis-specification of the death rate. Whilst optimal observation of the simple death process has received some attention, a full treatment of the uniqueness of the design has not been considered previously. Furthermore, no attention has been given to the time-constrained scenario. We have shown that the locally optimal design devised by Becker \& Kersting (1983) for the simple birth process can also be used to characterise the time-constrained optimal design for the simple death process. Moreover, their design specification greatly aids our understanding of both timeunconstrained and time-constrained designs for the simple death process.

We have focussed on giving useful information for practitioners, and showed that, in many instances, optimal equidistant designs can perform almost as well as the optimal design. This is likely to be of most benefit to practitioners using equidistant designs with limited observation opportunities, which may arise when there is significant cost associated with observation. We have also investigated logarithmic designs and shown that these can perform very well, but that equidistant designs tend to give slightly more information.

Our work here has focussed on experiments where organisms are subject to a constant hazard rate, and where the design is effectively static. There are several avenues for further investigation. We have already mentioned the possibility of allowing for hazard rates with specific functional forms. For example, if $\mu(t)=\mu t^{\beta}$ with $\beta(>0)$ known, so that lifetimes follow a Weibull distribution, we find that the optimal schedule for estimating $\mu$ is given by $t_{1}=\tau_{1}^{1 / \beta}$ and $t_{i}=\left(t_{i-1}^{\beta}+\tau_{i}\right)^{1 / \beta}$ for $i \geq 2$, where $\left(\tau_{i}\right)$ are precisely the optimal gaps exhibited earlier. It might also be possible to adapt the methods of Box \& Hunter (1965), which were developed for non-linear models, to obtain optimal or near-optimal sequential designs that would make use of information about the true parameter in order to improve the design as each observation is made. As a further direction, we are looking at optimal observation schedules for birth-death processes, including the SIS and other related models that arise in ecology and epidemiology.

Acknowledgement The first author acknowledges the support of the Australian Research Council Centre of Excellence for Mathematics and Statistics of Complex Systems, which funds his PhD scholarship. 


\section{References}

Atkinson, A. \& Donev, A. (1992), Optimum Experimental Designs, Clarendon Press.

Basawa, I. \& Prakasa Rao, B. (1980), Statistical Inference for Stochastic Processes, Academic Press.

Becker, G. \& Kersting, G. (1983), 'Design problems for the pure birth process', Advances in Applied Probability 15(2), 255-273.

Box, G. \& Hunter, W. (1965), 'The experimental study of physical mechanisms', Technometrics $7(1), 23-42$.

Box, G. \& Lucas, H. (1959), 'Design of experiments in non-linear situations', Biometrika 46(1-2), 77-90.

Chernoff, H. (1953), 'Locally optimal designs for estimating parameters', The Annals of Mathematical Statistics 24(4), 586-602.

Cook, A., Gibson, G. \& Gilligan, C. (2008), 'Optimal observation times in experimental epidemic processes', Biometrics 64(3), 860-868.

Corless, R., Gonnett, G., Hare, D., Jeffrey, D. \& Knuth, D. (1996), 'On the Lambert W function', Advances in Computational Mathematics 5, 329-359.

Ehrenfeld, S. (1962), 'Some experimental design problems in attribute life testing', Journal of the American Statistical Association 57(299), 668-679.

Fisher, R. (1922), 'On the mathematical foundations of theoretical statistics', Philosophical Transactions of the Royal Society of London, Series A 222, 309-368.

Ford, I., Titterington, M. \& Kitsos, C. (1989), 'Recent advances in nonlinear experimental design', Technometrics 31(1), 49-60.

Kleczkowski, A. \& Gilligan, C. (2007), 'Parameter estimation and prediction for the course of a single epidemic outbreak of a plant disease', Journal of the Royal Society Interface 4, 865-877.

Kuhn, H. \& Tucker, A. (1951), 'Nonlinear programming', Proceedings of 2nd Berkeley Symposium 222, 481-492.

Ogawa, J. (1962), 'Optimum spacing and grouping for the exponential distribution', In (Ed. Sarhan, A.E. and Greenberg, B.G.) Contributions to Order Statistics pp. 371-380.

Pronzato, L. \& Walter, E. (1985), 'Robust experiment design via stochastic approximation', Mathematical Biosciences 75(1), 103-120. 